\title{
Irradiation and anti-PD-L1 treatment synergistically promote antitumor immunity in mice
}

\author{
Liufu Deng, ${ }^{1}$ Hua Liang, ${ }^{1}$ Byron Burnette, ${ }^{1}$ Michael Beckett, ${ }^{1}$ \\ Thomas Darga, ${ }^{1}$ Ralph R. Weichselbaum, ${ }^{1}$ and Yang-Xin Fu² \\ 1Department of Radiation and Cellular Oncology, The Ludwig Center for Metastasis Research, and \\ 2Department of Pathology, University of Chicago, Chicago, Illinois, USA.
}

\begin{abstract}
High-dose ionizing irradiation (IR) results in direct tumor cell death and augments tumor-specific immunity, which enhances tumor control both locally and distantly. Unfortunately, local relapses often occur following IR treatment, indicating that IR-induced responses are inadequate to maintain antitumor immunity. Therapeutic blockade of the $\mathrm{T}$ cell negative regulator programmed death-ligand 1 (PD-L1, also called B7-H1) can enhance T cell effector function when PD-L1 is expressed in chronically inflamed tissues and tumors. Here, we demonstrate that PD-L1 was upregulated in the tumor microenvironment after IR. Administration of anti-PD-L1 enhanced the efficacy of IR through a cytotoxic T cell-dependent mechanism. Concomitant with IR-mediated tumor regression, we observed that IR and anti-PD-L1 synergistically reduced the local accumulation of tumor-infiltrating myeloid-derived suppressor cells (MDSCs), which suppress $T$ cells and alter the tumor immune microenvironment. Furthermore, activation of cytotoxic $T$ cells with combination therapy mediated the reduction of MDSCs in tumors through the cytotoxic actions of TNF. Our data provide evidence for a close interaction between IR, T cells, and the PD-L1/PD-1 axis and establish a basis for the rational design of combination therapy with immune modulators and radiotherapy.
\end{abstract}

\section{Introduction}

Radiotherapy (RT) is widely used in the treatment of primary and metastatic tumors. The biological responses of tumors to radiation include DNA damage, modulation of signal transduction, and alteration of the inflammatory tumor microenvironment. Recent studies from our laboratory and others have revealed that high-dose ablative radiation, given in 1 to 3 fractions, can trigger adaptive immune responses that mediate tumor regression (1-3). During the inflammatory response that occurs after radiation, tumors may develop multiple resistance mechanisms that facilitate tumor relapse (4). Little is known about how ionizing irradiation (IR) or IR-mediated immune responses alter the tumor microenvironment and what host pathways modulate the strength or duration of IR-induced $\mathrm{T}$ cell responses.

The tumor microenvironment is populated by various types of inhibitory immune cells including Tregs, alternatively activated macrophages, and myeloid-derived suppression cells (MDSCs), which suppress $\mathrm{T}$ cell activation and promote tumor outgrowth (5). Recent studies indicate that MDSCs also play an essential role in chemoresistance and radioresistance. In particular, the production of CXCL $1 / 2$ by breast cancer cells has been reported to attract MDSCs, which in turn secrete S100A8/9 proteins that function as prosurvival factors and rescue cancer cells from the cytotoxic effects of chemotherapy (6). Thus, MDSCs augment the resistance of cancer cells to cytotoxic therapies both directly, by promoting tumor cell survival, and indirectly, by inhibiting the antitumor $\mathrm{T}$ cell response. While it is well documented that MDSCs can negatively regulate $\mathrm{T}$ cell function, other evidence suggests that $\mathrm{T}$ cells

Authorship note: Liufu Deng and Hua Liang contributed equally to this work. Conflict of interest: The authors have declared that no conflict of interest exists. Citation for this article: J Clin Invest. 2014;124(2):687-695. doi:10.1172/JCI67313. might act to counterregulate MDSCs (7). Therapeutic blockade of immune checkpoints has been associated with a reversal in the distribution and proportion of MDSCs $(8,9)$. In addition, a reduction in circulating MDSCs was associated with regression of metastatic tumors in a melanoma patient treated with ipilimumab and radiotherapy (10). Aside from these correlative data, a complete understanding of how immune checkpoint inhibitors might disable the immune suppressive activity of MDSCs in combination with RT or chemotherapy is lacking.

The PD-L1/PD-1 axis has been characterized as a potent inhibitor of immune activation, particularly through inhibition of effector T cell function (11). The PD-L1 (also called B7-H1) protein is undetectable in most normal tissues and is inducible in various cell types by inflammatory cytokines, especially type I and type II IFNs (12-15). Evidence for a tissue-protective role of PD-L1 is revealed through the association of upregulated PD-L1 expression and amelioration of autoimmunity in several models, such as EAE and autoimmune diabetes $(16,17)$. In contrast, some viruses can induce PD-L1/PD-1 signaling to escape the host immune response by inducing $T$ cell exhaustion, which results in chronic infection (18-20). Proinflammatory cytokines have been reported to be substantially elevated in the tumor microenvironment, and elevated expression is correlated with tumor progression (21). PD-L1 expression has also been observed in a wide variety of solid malignancies, suggesting that PD-L1 may be a dominant mechanism of immune suppression (22). Moreover, inhibitors of the PD-L1/ PD-1 axis have been reported to generate potent antitumor activity in murine tumor models and clinical trials (23-26). Supporting a dominant role for PD-L1 in local immune suppression within the tumor microenvironment, patients lacking PD-L1 expression in tumor biopsies did not responded to anti-PD-1 antibody treatment, while a high percentage of patients with PD-L1+ tumors did 
A

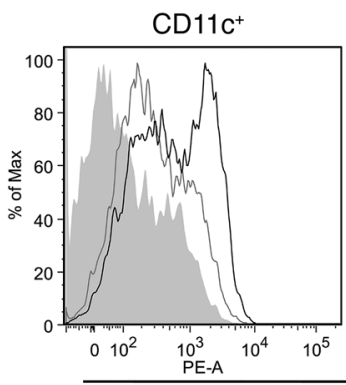

$\mathrm{CD}_{11 \mathrm{~b}} \mathrm{Gr1}^{+}$

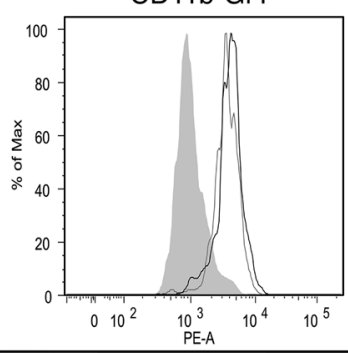

$\mathrm{CD} 1 \mathrm{~b}^{+} \mathrm{F} 4 / 80^{+}$

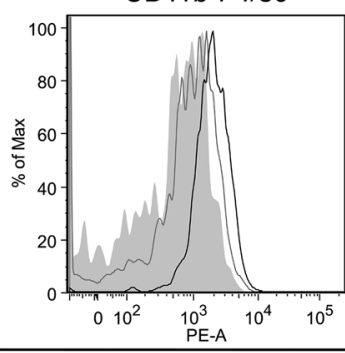

CD45

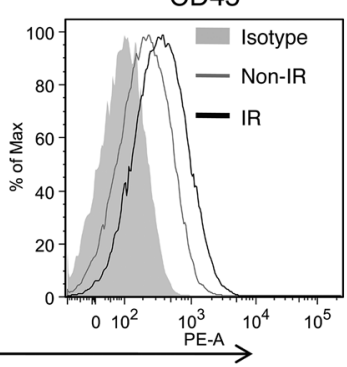

PD-L1

B

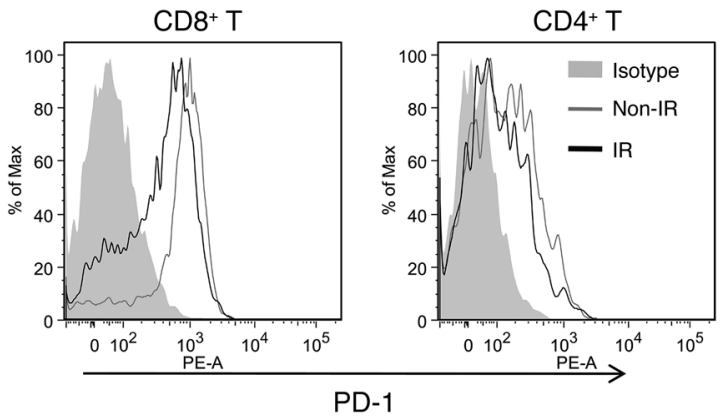

\section{Figure 1}

The profile of PD-L1 and PD-1 expression in tumor microenvironments is altered after IR. BALB/c mice were injected s.c. into the flank with $1 \times 10^{6}$ TUBO cells. On day 14, mice were locally treated with one 12-Gy dose of IR. Three days after IR, tumors were removed and digested into singlecell suspensions, which were blocked with anti-FcR mAbs and then subjected to surface staining. PD-L1 expression on myeloid cells and tumor cells (A) and PD-1 expression on T cells (B). Representative data are shown from three (A and $\mathbf{B})$ experiments conducted using 3 mice per group.

respond to the treatment (25). We hypothesized that IR induces a local inflammatory response that could enhance the infiltration of tumor-specific T cells and simultaneously induce PD-L1 expression in the tumor microenvironment that markedly weakens IR-induced antitumor immunity. The concept of IR-induced PD-L1 expression and subsequent blockade might broaden the application of PD-L1/PD-1 axis inhibitors and prove to be a potent anticancer therapy when combined with RT.

Here, we report that local upregulation of the PD-L1/PD-1 axis following IR suppresses radiation-induced immune responses, thereby limiting the full expression of antitumor immunity and facilitating relapse. Combination therapy with IR and PD-L1 blockade optimizes antitumor immunity and consequently leads to the elimination of MDSCs through enhanced production of T cell-derived TNF. Therefore, this study provides insight into the rational design of combination therapies involving anti-PD-L1 and RT to improve responses in cancer patients.

\section{Results}

Increased PD-L1 expression in tumor tissue following IR. Localized ablative radiation has been shown to mediate tumor regression in a $T$ cell-dependent fashion (3). In addition, the production of type I IFN has been demonstrated to be an essential pathway regulating radiation-induced antitumor immunity (2). Despite the immunestimulating effects of radiation, relapses often occur, suggesting that radiation likely does not optimally engage adaptive immunity to mediate complete tumor elimination. In part, incomplete tumor eradication by radiation-induced adaptive immunity could be due to the engagement of $\mathrm{T}$ cell-negative regulatory pathways such as the PD-L1/PD-1 axis. To investigate whether IR induces
PD-L1 upregulation in the tumor microenvironment, we treated TUBO tumors with 12 Gy and removed the tumor tissue 3 days after radiation to conduct flow cytometric analysis of PD-L1 expression on cells in the tumor microenvironment. We analyzed PD-L1 expression on tumor cells (CD45-), DCs (CD11c $)$, MDSCs $\left(\mathrm{CD} 11 \mathrm{~b}^{+} \mathrm{Gr} 1^{+}\right)$, and macrophages $\left(\mathrm{CD} 11 \mathrm{~b}^{+} \mathrm{F} 4 / 80^{+}\right)$. An increase in the expression of PD-L1 was observed on DCs and tumor cells after radiation compared with expression levels in the same cell populations in nonirradiated control tumors (Figure 1A). PD-L1 expression was also slightly elevated on macrophages (Figure 1A). Expression of PD-L1 on MDSCs did not change following IR; however, MDSCs from untreated tumors had high baseline PD-L1 expression levels (Figure 1A). The expression of PD-1 was determined on $\mathrm{CD}^{+} \mathrm{T}$ cells and $\mathrm{CD}^{+} \mathrm{T}$ cells. Both $\mathrm{T}$ cell subsets express PD- 1 in the tumor microenvironment, and $\mathrm{CD}^{+} \mathrm{T}$ cells expressed a more uniformly high level of PD-1. The level of $\mathrm{PD}-1$ was slightly downregulated on $\mathrm{CD}^{+} \mathrm{T}$ cells on day 3 after IR (Figure 1B), despite the increased presence of its ligand in the environment. These data indicate that alteration of the PD-L1/PD-1 axis in the tumor microenvironment might inhibit $\mathrm{T}$ cell function and result in tumor relapse. Furthermore, these data raise the possibility that negative regulation of tumor-infiltrating $\mathrm{T}$ cells through PD-L1/PD-1 might be an important host-mediated mechanism of acquired radioresistance in tumors.

Synergistic effect of IR and PD-L1 blockade in antitumor immunity. PD-L1 expression in the tumor microenvironment has been associated with poor outcomes following chemoradiotherapy in cancer patients $(27,28)$. Conversely, IR-induced increases in tumor-infiltrating lymphocytes (TILs) and upregulation of PD-L1 could provide an opportunity for PD-L1 blockade that would uncover the 

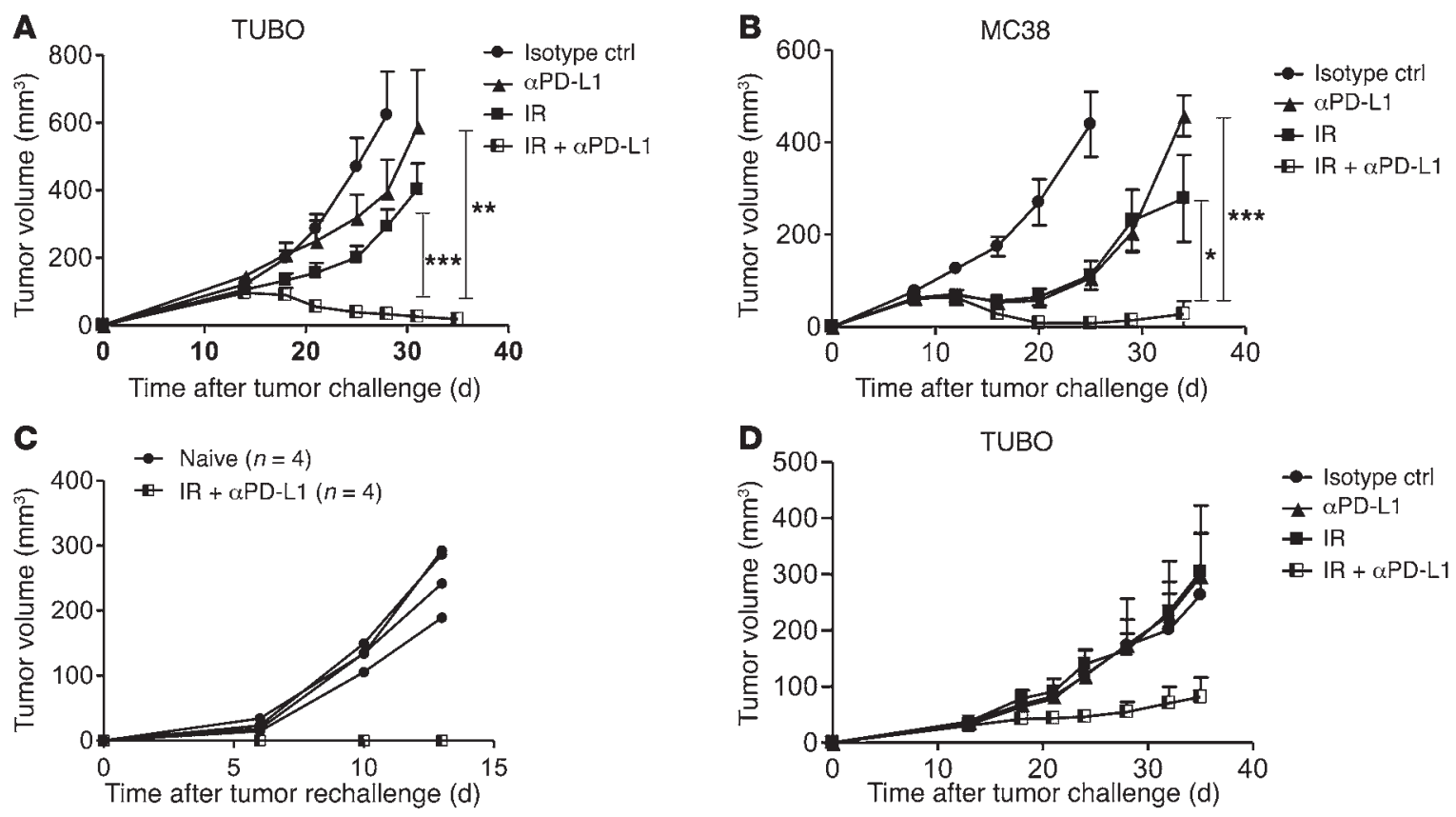

\section{Figure 2}

IR and PD-L1 blockade synergistically amplify the antitumor effect. (A) Combination of anti-PD-L1 ( $\alpha$ PD-L1) and IR significantly enhanced the inhibition of TUBO tumor growth. BALB/c mice were inoculated s.c. on day 0 with $1 \times 10^{6}$ TUBO cells. Tumors locally received one $12-G y$ dose on day 14 and/or $200 \mu \mathrm{g}$ anti-PD-L1 (clone 10F.9G2) or isotype control i.p. every three days for a total of four times. ${ }^{* *} P<0.01$; ${ }^{* * *} P<0.001$. (B) Combination therapy greatly delayed MC38 tumor growth compared with single treatments. C57BL/6 mice were injected s.c. on day 0 with $1 \times 10^{6}$ MC38 cells. Tumors received 20 Gy on day 8 , and antibodies were started on day 8 and administered as described in A. ${ }^{*} P<0.05$; ${ }^{* * *} P<0.001$. (C) Tumor-free mice that underwent combination therapy were resistant to the tumor rechallenge. Thirty days after tumor eradication, the mice treated as in A were rechallenged with $2 \times 10^{6}$ TUBO cells on the opposite flank. (D) Systemic effect of combination treatment greatly reduced the growth of secondary tumors. TUBO tumors on the right flank were treated with 12 Gy or anti-PD-L1 alone, or with 12 Gy plus antiPD-L1, as described in A. Tumors on the left flank were measured and monitored. Representative data are shown from three (A) or two (B-D) experiments conducted with 6 to 8 (A and D), 5 (B), or $4(\mathbf{C})$ mice per group.

full cytotoxic potential of host immunity against the tumor. We hypothesized that blockade of PD-L1 enhances RT by alleviating the inhibitory action of PD-L1 on T cells. To test this hypothesis, TUBO cells were implanted, and 14 days later tumors were treated with IR (12 Gy), anti-PD-L1 alone, or IR plus anti-PD-L1. Anti-PD-L1 by itself had a slight impact on tumor growth, whereas RT slowed tumor progression. Treatment with a combination of IR plus antiPD-L1 effectively controlled tumor growth $(P=0.0022$, anti-PD-L1 vs. IR plus anti-PD-L1 $=587.3 \pm 169.1 \mathrm{~mm}$ vs. $25.59 \pm 10.26 \mathrm{~mm}$ on day $31 ; P=0.0002$, IR vs. IR plus anti-PD-L1 $=402.8 \pm 76.73 \mathrm{~mm}$ vs. $25.59 \pm 10.26 \mathrm{~mm}$ on day 31 ) (Figure 2A). The effectiveness of the combination treatment was also confirmed in the distinct syngeneic colon cancer model MC38 $(P<0.0001$, anti-PD-L1 vs. IR plus anti$\mathrm{PD}-\mathrm{L} 1=457.6 \pm 44.24 \mathrm{~mm}$ vs. $27.85 \pm 27.85 \mathrm{~mm}$ on day $34 ; P=0.034$, IR vs. IR plus anti-PD-L1 = 278.6 $\pm 94.20 \mathrm{~mm}$ vs. $27.85 \pm 27.85 \mathrm{~mm}$ on day 34) (Figure 2B).

To address whether combination therapy resulted in the generation of prolonged protective $T$ cell immunity, 30 days after complete tumor rejection, mice were rechallenged with a much higher dose $\left(2 \times 10^{6}\right.$ cells $)$ of TUBO tumor cells on the opposite flank. No palpable tumors were detected on the treated mice after a few weeks, whereas tumors on naive mice were palpable after 1 week (Figure 2C). The capacity of local radiation to mediate effects on the tumor outside the radiation field, termed the abscopal effect, has been observed in several types of human cancers, including melanoma and renal cell carcinoma $(29,30)$. Although, the abscopal effect has not been formally linked to host immune responses, several reports have observed abscopal regression in patients undergoing RT and immunotherapy, suggesting that host immunity could be a major determinant (10). We tested whether combination treatment with IR plus anti-PD-L1 could exert abscopal effects on secondary tumors that did not receive local IR. To test this, tumor cells were implanted in both flanks. Tumors intended for treatment received 5 times more cells than tumors on the contralateral flank that would serve as abscopal tumors ( $10^{6}$ cells vs. $2 \times 10^{5}$ cells). The primary tumors received treatments, as shown in Figure 2A. A growth delay was observed in the IR plus anti-PD-L1 combination group, but not in groups receiving either treatment alone (Figure 2D). These results suggest that anti-PD-L1 treatment not only improves the effects of IR on the primary tumor, but also mediates an abscopal effect on distant tumors.

$C D 8^{+} T$ cells are essential for the efficacy of combination therapy. To investigate the importance of $\mathrm{CD}^{+} \mathrm{T}$ cells in combination therapy, $\mathrm{CD} 8^{+}$ $\mathrm{T}$ cells were depleted using antibodies in the mice treated with IR plus anti-PD-L1. Depletion of $\mathrm{CD}^{+} \mathrm{T}$ cells completely abolished the effectiveness of the combination treatment, resulting in rapid tumor outgrowth (Figure $3 \mathrm{~A}, P=0.025$ on day 32 ). In contrast, depletion of $\mathrm{CD}^{+} \mathrm{T}$ cells did not change tumor growth after the combination treatment (Supplemental Figure 1; supplemental material available online with this article; doi:10.1172/JCI67313DS1). 
A

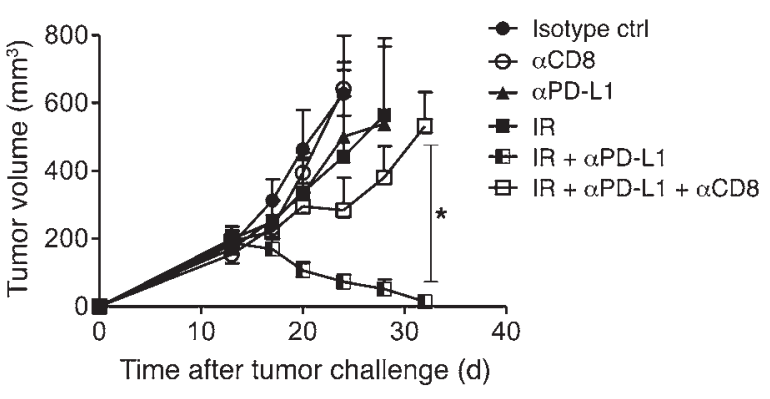

B

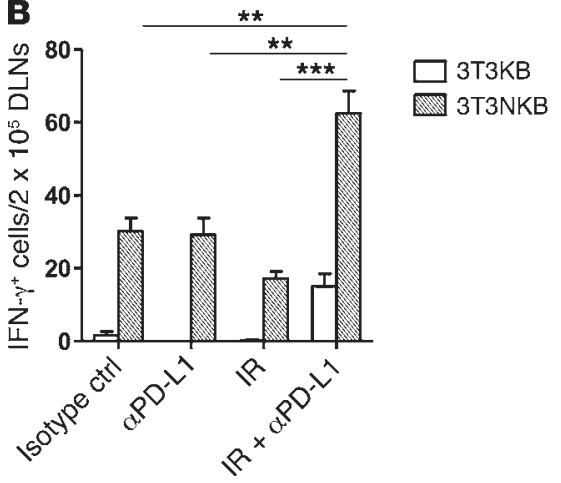

\section{Figure 3}

$\mathrm{CD}^{+} \mathrm{T}$ cells are required for the efficacy of IR and anti-PD-L1 combination treatment. (A) Tumor regression by combination treatment with antiPD-L1 and IR was mediated by CD8 ${ }^{+}$T cells. Tumors received $12 \mathrm{~Gy}$ and mice were treated with anti-PD-L1, as described in Figure 2A. Starting from 1 day before IR, $250 \mu \mathrm{g}$ of depletion antibodies against CD8 ${ }^{+} \mathrm{T}$ cells (clone 2.43) was injected i.p. every 3 days for a total of four times. ${ }^{*} P<0.05$. (B) Combination therapy greatly enhanced the antigen-specific response of CD8 ${ }^{+} \mathrm{T}$ cells. TUBO tumors received 12 Gy, and mice were treated with anti-PD-L1 as described in Figure 2A. Nine days after IR, the draining LNs were removed and subjected to ELISPOT assays. ${ }^{\star \star} P<0.01 ;{ }^{* *} P<0.001$. Representative data are shown from two $(\mathbf{A})$ and three (B) experiments conducted with 5 to 6 (A) or 4 (B) mice per group.

Altogether, these results demonstrate that $\mathrm{CD}^{+} \mathrm{T}$ cells are necessary for the therapeutic effects of IR plus anti-PD-L1 therapy and are in agreement with our previous observations using IR as a single modality $(2,3,31)$. Next, we hypothesized that the combination of IR and anti-PD-L1 treatment enhances tumor antigen-specific $T$ cell responses. We used a sensitive system to detect HER2/neu-specific $\mathrm{CD}^{+} \mathrm{T}$ cells in the TUBO model that has been described in previous work (32). Briefly, 3T3 fibroblasts were used as APCs by stable expression of the MHC-I molecule H2- $\mathrm{K}^{\mathrm{d}}$ and B7.1 (3T3KB). To allow presentation of endogenously generated HER2/neu peptides, the $3 \mathrm{~T} 3 \mathrm{~KB}$ cells were transfected with full-length rat neu (3T3NKB), which allows for measurement of neu-specific $\mathrm{CD}^{+} \mathrm{T}$ cell responses with proper negative control antigens. Nine days after IR, draining LNs from tumor-bearing mice were removed, and an IFN- $\gamma$ ELISPOT assay was performed using the 3T3 APC system. The number of neu-specific IFN- $\gamma$-producing T cells was significantly increased in the tumor-draining LNs of mice that received combination treatment compared with those that received IR or anti-PD-L1 alone $(P=0.0015$, anti-PD-L1 vs. IR plus anti-PD-L1; $P<0.0001$, IR vs. IR plus anti-PD-L1) (Figure $3 B$ ). Our results indicate that IR plus anti-PD-L1 therapy improves tumor control by enhancing host tumor antigen-specific $\mathrm{T}$ cell function in the tumor microenvironment and by enhancing the systemic activation of tumor-specific $T$ cells in secondary lymphoid organs.

$I R$ and anti-PD-L1 combination therapy reduce the accumulation of MDSCs. MDSCs have been correlated with radioresistance, and MDSC accumulation in the tumor microenvironment might promote tumor relapse through direct effects on tumor cell survival and via indirect effects on local $\mathrm{T}$ cell suppression $(10,33)$. The reports have been mixed regarding the capacity of MDSCs to exert $\mathrm{T}$ cell suppression, and the discrepancies are likely due to differences in the tumor models and phenotypic differences in MDSC subsets (34). To determine the $\mathrm{T}$ cell-suppressive capacity of MDSCs in our animal models, we used positive selection of $\mathrm{Gr} 1^{+}$ cells to obtain MDSCs. The resulting purified $\mathrm{Gr} 1^{+}$cells were subjected to a $\mathrm{T}$ cell suppression assay. Our data show that $\mathrm{Gr} 1^{+}$cells greatly suppress the proliferation of $\mathrm{CD}^{+} \mathrm{T}$ cells, confirming the immune-suppressive effects of these cells (Supplemental Figure 2).
To determine the dynamics of MDSC accumulation in the tumor microenvironment, we sought to investigate possible changes in the MDSC population following treatment. On day 10 after IR, we observed that MDSCs, defined by $\mathrm{CD} 45^{+} \mathrm{CD} 11 \mathrm{~b}^{+} \mathrm{Gr} 1^{+}$expression, were reduced by IR and/or anti-PD-L1 (Figure 4A). In tumors that received anti-PD-L1 or IR treatment alone, the percentage of MDSCs in the total $\mathrm{CD} 45^{+}$cell population decreased from $19.58 \% \pm 3.66 \%$ in untreated tumors to $7.33 \% \pm 2.22 \%(P=0.016)$ and $4.78 \% \pm 2.49 \%(P=0.0074)$, respectively (Figure $4 \mathrm{~B}$, left). Combination therapy with anti-PD-L1 and IR exhibited the greatest effect on MDSCs and further reduced the percentage to $0.38 \% \pm 0.16 \%$ of total $\mathrm{CD} 45^{+}$cells $(P<0.0001$, IR plus anti-PD-L1 vs. isotype control) (Figure 4B, left). The percentages of macrophages $\left(\mathrm{CD} 11 \mathrm{~b}^{+} \mathrm{F} 4 / 80^{+}\right), \mathrm{CD}^{+} \mathrm{T}$ cells, and $\mathrm{CD} 4^{+} \mathrm{T}$ cells were unaffected by either treatment alone or by combination treatment (Figure 4B, left). We found that combination therapy or either single treatment did not mediate significant changes in the population of Tregs (Supplemental Figure 3). The extent of local reduction in MDSCs was associated with enhanced tumor growth delay and tumor regression. These results raise the possibility that a local reduction in MDSCs is an essential component in the therapeutic efficacy of combination treatment with local IR and anti-PD-L1.

Two possible mechanisms might explain the observed reduction in MDSCs after combination treatment: decreased trafficking of MDSCs to the tumor or increased MDSC cell death in situ. To determine the potential contribution of these two mechanisms, we first examined the percentage of MDSCs at an earlier time, day 3 after IR, to examine the kinetics in more detail. On day 3 after IR, there was no change in the percentage of MDSCs in tumors after single or combination treatment, indicating that the treatment does not affect the recruitment of MDSCs (Figure 4B, right). It is noteworthy that the percentage of $\mathrm{CD}^{+} \mathrm{T}$ cells in tumors was significantly decreased after IR alone, suggesting that local radiation likely eliminates some proportion of the TILs that are present at the time of treatment and that newly infiltrating $\mathrm{CD}^{+} \mathrm{T}$ cells replenish the population over time. Infiltration of new $\mathrm{CD}^{+}$ $T$ cells following IR might also explain the reduced expression of PD-1 on tumor-infiltrating $\mathrm{CD}^{+} \mathrm{T}$ cells that we previously 
A

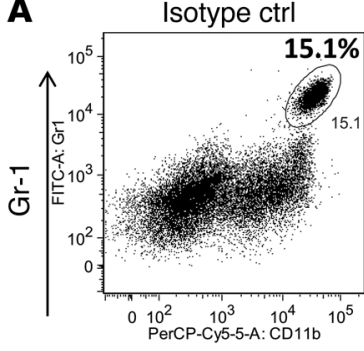

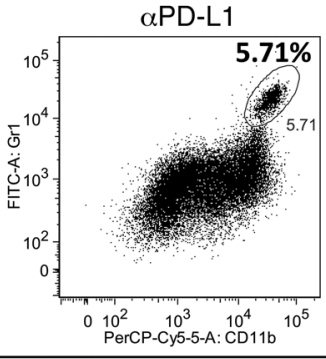

aPD-L1

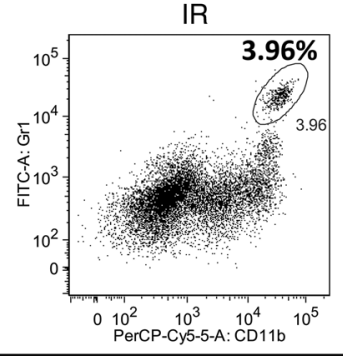

IR

CD11b
B

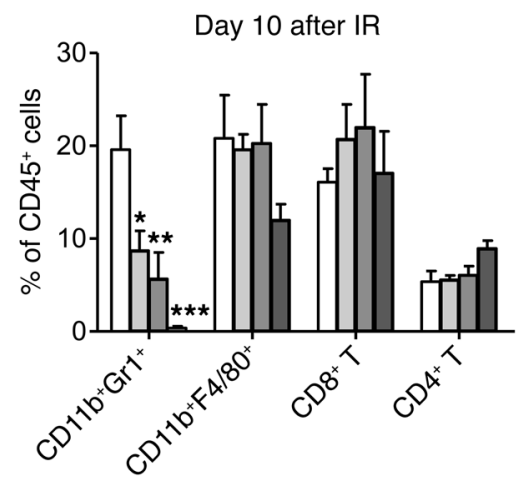

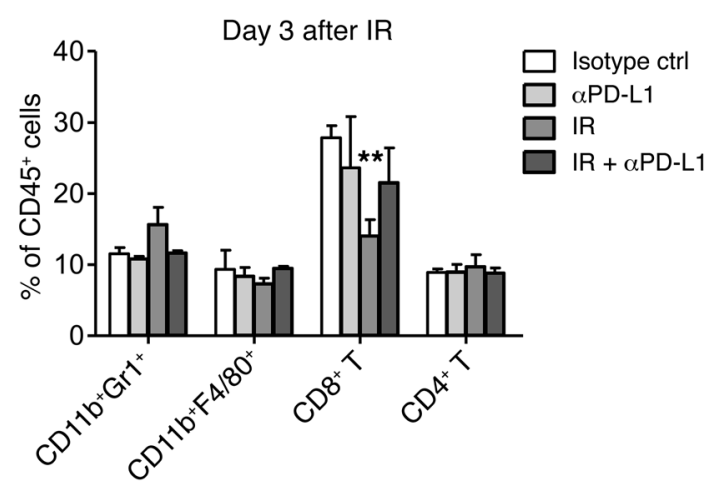

Figure 4

IR and PD-L1 blockade induce the reduction of MDSCs. Tumors received $12 \mathrm{~Gy}$, and mice were treated with anti-PD-L1 as described in Figure 2A. Three days or 10 days after IR, tumors were removed to obtain cell suspensions for surface staining. (A) Flow cytometric analysis of MDSCs (CD11b+Gr1+) gated on $\mathrm{CD}_{45}{ }^{+}$cells in tumors 10 days after IR. (B) Quantitative data of the percentage of MDSCs $\left(\mathrm{CD} 11 \mathrm{~b}+\mathrm{Gr} 1^{+}\right)$, macrophages (CD11b+F4/80+), CD8 ${ }^{+} \mathrm{T}$ cells, and CD4 ${ }^{+} \mathrm{T}$ cells relative to $\mathrm{CD} 45^{+}$cells on day 10 (left) and on day 3 (right) after IR. ${ }^{\star} P<0.05 ;{ }^{* \star} P<0.01$; ${ }^{* * \star} P<0.001$. Representative data are shown from two (A and $\mathbf{B}$ ) experiments conducted with 5 mice per group. observed after IR (Figure 1B). To determine whether the effect of IR on MDSCs was only specific to the tumor, we examined the proportion of immune cell populations in the periphery after IR. On day 10 after IR, combination treatment, but neither of the single treatments alone, resulted in a decrease in splenic MDSCs, while the percentages of macrophages, DCs $\left(\mathrm{CD} 11 \mathrm{c}^{+}\right), \mathrm{B}$ cells $\left(\mathrm{B} 220^{+}\right)$, $\mathrm{CD}^{+} \mathrm{T}$ cells, and $\mathrm{CD} 4^{+} \mathrm{T}$ cells were unaffected (Supplemental Figure 4, left). Similar to the kinetics of MDSC disappearance in the tumor, we observed no difference in the percentage of splenic MDSCs on day 3 after IR (Supplemental Figure 4, right). Together, these data indicate that both the local and systemic reductions in MDSCs are associated with enhanced $T$ cell functional activity and that a reduction in MDSCs occurs with a delay in kinetics.

Reduced accumulation of MDSCs following combination therapy is dependent on $C D 8^{+} T$ cells. We next sought to determine whether IR kills MDSCs directly or whether $\mathrm{CD}^{+}$cells directly contribute to the decreased proportion of MDSCs following combination therapy. We stained tumor sections derived from untreated control and IR plus anti-PD-L1 mice with antibodies against CD11b, $\mathrm{Gr} 1, \mathrm{CD} 8$, and activated caspase 3 . In addition to the reduction in $\mathrm{Gr}^{+}$cells in the combination-treated tumors, a profound difference in the colocalization of remaining $\mathrm{Gr}^{+}$and $\mathrm{CD}^{+}$cells was also revealed (Figure 5A). To quantify the degree of colocalization of $\mathrm{Gr}^{+}$and $\mathrm{CD}^{+}$cells, we measured the average distance between cells staining positive for each marker. The average distance between a CD $11 \mathrm{~b}^{+} \mathrm{Gr} 1^{+}$and an adjacent $\mathrm{CD}^{+} \mathrm{T}$ cell was significantly reduced in tumors treated with IR plus anti-PD-L1 compared with that observed in untreated tumors (Figure 5B, $P<0.01)$. In tumors treated with combination therapy, we also observed elevated levels of activated caspase 3 in $\mathrm{Gr} 1^{+}$cells that were closely associated with $\mathrm{CD}^{+} \mathrm{T}$ cells (insets of Figure $5 \mathrm{~A}$ ). These results suggest that $\mathrm{CD}^{+}$cells is directly involved in controlling MDSC cells by inducing apoptosis of MDSCs. To begin to address this possibility, we examined changes in the proportion of MDSCs after depletion of CD8 ${ }^{+} \mathrm{T}$ cells in combination treatment.
Our results indicate that depleting $\mathrm{CD}^{+} \mathrm{T}$ cells restored the levels of MDSCs to those observed in untreated control mice (Figure 5, $\mathrm{C}$ and $\mathrm{D}$ ). These results further solidify the association of $\mathrm{CD}^{+}$ $\mathrm{T}$ cells with local accumulation of MDSCs; however, these data could not definitively determine a direct mechanistic relationship.

Restoration of the proportions of MDSCs in the combination treatment following $\mathrm{CD}^{+} \mathrm{T}$ cell depletion $(P=0.0038)$ raised the possibility that $\mathrm{CD}^{+} \mathrm{T}$ cells are directly involved in limiting the accumulation of MDSCs in the tumor microenvironment by mediating MDSC death. We investigated this possibility by coculturing MDSCs derived from the spleens of tumor-bearing mice with activated $\mathrm{CD}^{+} \mathrm{T}$ cells. Coculture of activated T cells with MDSCs resulted in an increase (from $11.75 \% \pm 0.48 \%$ to $44.38 \% \pm 0.63 \%$ ) in annexin $\mathrm{V}^{+}$(apoptotic) MDSCs compared with those cultured with resting $\mathrm{CD}^{+} \mathrm{T}$ cells (Figure $6, \mathrm{~A}$ and $\mathrm{B}$ ). In addition, we found that expression of PD-L1 protected MDSCs from cell death induced by activated $\mathrm{CD}^{+} \mathrm{T}$ cells (data not shown). These data raise the possibility that $\mathrm{RT}$ and anti-PD-L1 combination therapy restores the function of $\mathrm{CD}^{+} \mathrm{T}$ cells, which, in turn, results in the direct elimination of MDSCs.

Because polyclonal activated T cells mediated MDSC apoptosis in our in vitro assay, we hypothesized that the interaction takes place in an antigen-nonspecific manner. We further hypothesized that $\mathrm{T}$ cell-derived cytokines are involved in the induction of MDSC apoptosis. Compared with blockade of IFN- $\gamma$, we observed that neutralization of TNF in the coculture system significantly reduced the fraction of annexin $\mathrm{V}^{+}$MDSCs (Figure 6, $\mathrm{A}$ and $\mathrm{B}$ ). Nevertheless, we did not observe a synergy between TNF and IFN- $\gamma$ with induction of MDSC apoptosis (Figure 6, A and B). Next, we asked whether TNF or IFN- $\gamma$ is sufficient to induce MDSC death in the absence of T cells. Treatment of MDSCs with TNF induced high levels of apoptosis (30\%) at a concentration of $50 \mathrm{ng} / \mathrm{ml} \mathrm{com-}$ pared with those seen with the background (10\%), whereas IFN- $\gamma$ induced up to $16 \%$ annexin $\mathrm{V}^{+}$apoptosis in MDSCs at a concentration of $20 \mathrm{ng} / \mathrm{ml}$ (Figure 6C). These results show that MDSCs 
A

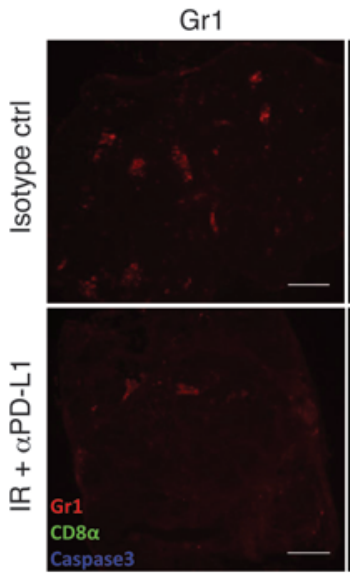

C

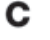

Isotype ctrl

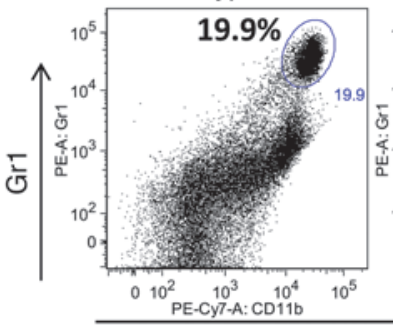

CD8

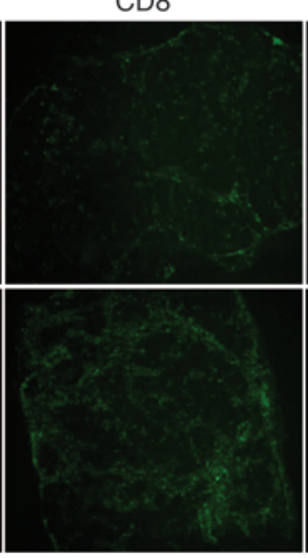

$\mathrm{IR}+\alpha \mathrm{PD}-\mathrm{L} 1$

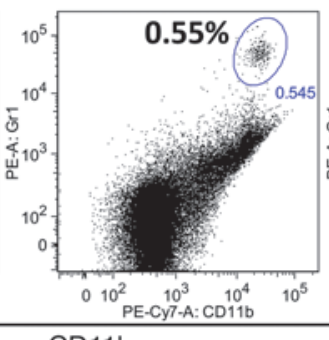

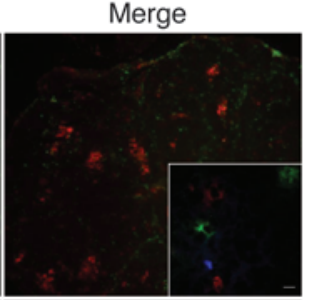

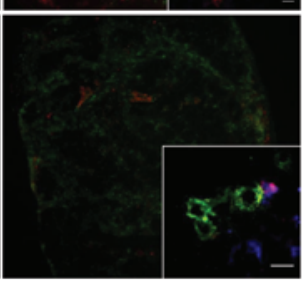

$\mathrm{IR}+\alpha \mathrm{PD}-\mathrm{L} 1+\alpha \mathrm{CD} 8$

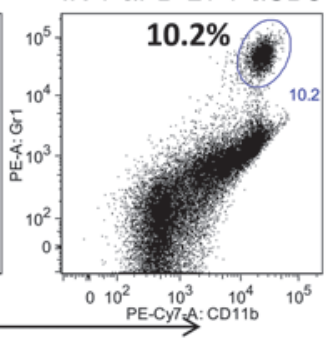

B

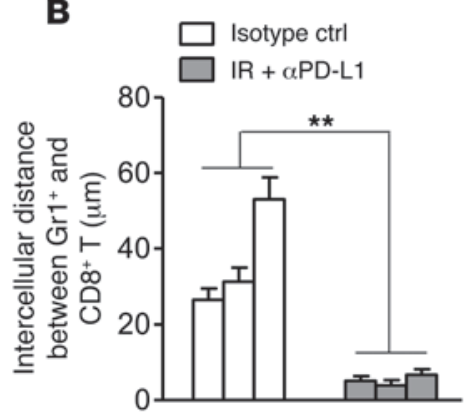

D

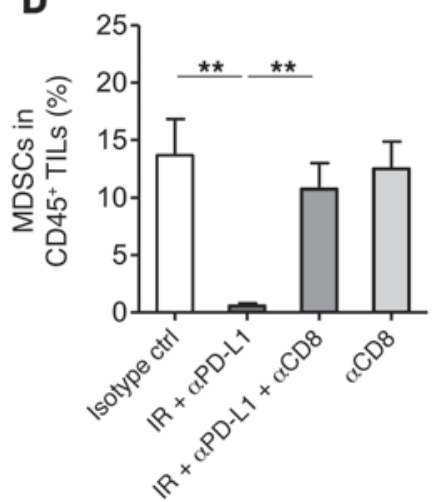

Figure 5

CD8 ${ }^{+} \mathrm{T}$ cells mediate the reduction of MDSCs in IR and anti-PD-L1 combination treatment. TUBO tumor-bearing mice were treated with IR and antibodies as described in Figure 2A and Figure 3A. Ten days after IR, the tumors were removed. (A) Immunofluorescence staining of frozen tumor sections. Top row, untreated tumor; Bottom row, tumor treated with IR and anti-PD-L1. Scale bars: $100 \mu \mathrm{m}$; original magnification, $\times 4$. Inset scale bars: $5 \mu \mathrm{m}$; original magnification, $\times 100$. (B) Quantification of distance from CD11 b+Gr1+ cells to the closest CD ${ }^{+} T$ cell in a high-power field $(\times 40)$. Fifteen to eighteen high-power fields were counted for each section. ${ }^{* \star} P<0.01$. Sections were obtained from three tumors per group. The quantification performed on the individual tumor from each group is shown in each histogram. (C) Representative dot plots of MDSCs gated on a CD45+ cell population. (D) The reduction of the proportion of MDSCs with combination therapy was rescued after the depletion of CD8 ${ }^{+} \mathrm{T}$ cells. ${ }^{\star \star} P<0.01$. Representative data are shown from two $(\mathbf{A}-\mathbf{D})$ experiments conducted with $3(\mathbf{A}$ and $\mathbf{B})$ or $4(\mathbf{C}$ and $\mathbf{D})$ mice per group.

are more sensitive to TNF-mediated cell death. To begin to assess the role of local TNF production in MDSC viability in vivo, established tumors were locally injected with adenoviral vector TNF (Ad-TNF) or Ad-LacZ to drive local expression of TNF in the tumor microenvironment. Exogenous TNF expression from the adenoviral vector significantly decreased MDSCs in vivo compared with the effects of control Ad-LacZ (Supplemental Figure 5). These results indicate that TNF can directly induce MDSC death both in vitro and in vivo and that activated $\mathrm{T}$ cells could be the essential source of TNF during combination treatment.

To confirm whether TNF is necessary for the reduction of MDSCs after combination treatment, we conducted in vivo neutralization experiments. When TNF was neutralized in mice receiving IR plus anti-PD-L1 combination therapy, tumor regression was significantly impaired (Figure 6D). To determine the therapeutic significance of a reduced accumulation of MDSCs in the combination therapy group, we performed in vivo depletion of residual MDSCs in MC38 tumor-bearing mice that received IR alone. Since Ly-6C is expressed on both MDSCs and activated $\mathrm{CD}^{+} \mathrm{T}$ cells, anti-Gr1-depleting antibody (Ly-6C/Ly-6G) (clone RB6-8C5) could potentially deplete MDSCs and activated CD8 ${ }^{+}$ $\mathrm{T}$ cells, rendering conflicting results. Considering this, we chose the anti-Ly-6G (clone 1A8) antibody, which specifically targets MDSCs, for the depletion experiments. Antibody-mediated depletion of MDSCs enhanced the effect of local IR and recapitulated the therapeutic benefit of combination treatment with IR and anti-PD-L1 (Figure 6E). These results suggest that MDSCs remaining after local IR can prevent complete $T$ cell-mediated regression and that the reduction of MDSCs may be one of the mechanisms underlying the efficacy of IR plus anti-PD-L1 combination treatment. Taken together, our results indicate that combination therapy of IR and anti-PD-L1 can enhance the activation of $\mathrm{CD}^{+} \mathrm{T}$ cells, an effect that negatively regulates the accumulation of MDSCs by TNF and facilitates tumor regression.

\section{Discussion}

Localized ablative IR has been shown to mediate tumor regression in a $T$ cell-dependent and IFN- $\beta$-dependent fashion $(2,3,31)$. Type I IFNs can induce PD-L1 expression, which is proposed to limit local immunity and promote tumor relapse. PD-L1 expression in the tumor microenvironment provides an opportunity for therapeutic intervention using regulators such as anti-PD-L1 and anti-PD-1. Clinical trials demonstrated that PD-L1 or PD-1 antagonistic antibodies can elicit responses in $15 \%-25 \%$ patients, depending on the tumor type, and that the presence of PD-L1 is a biomarker for success of the treatment $(24,25)$. Human tumors can also respond to ablative RT, although this strategy has not been uniformly successful due to local or distant failures $(35,36)$. 

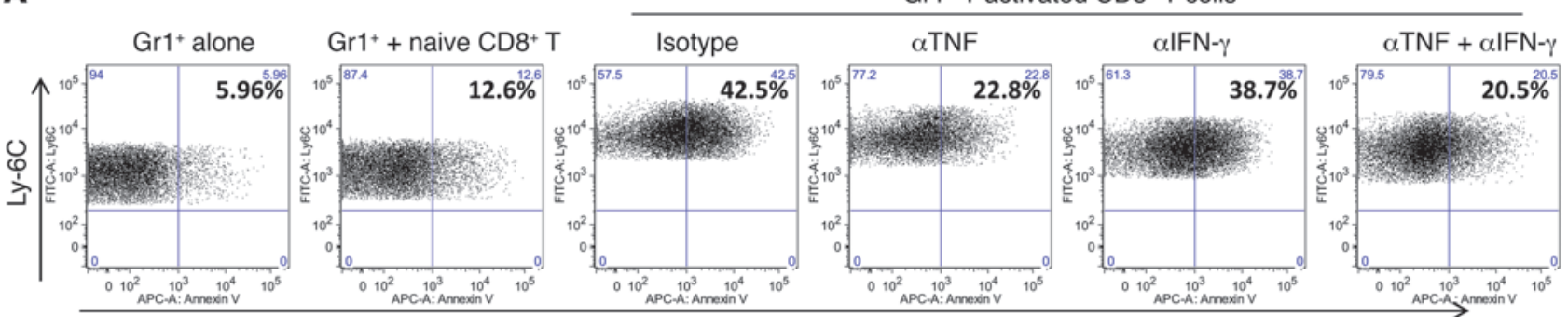

Annexin V

B
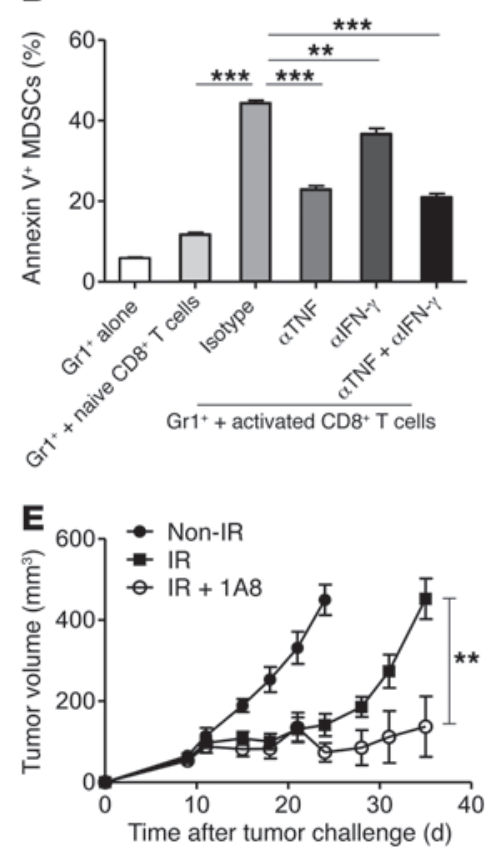

\section{c}

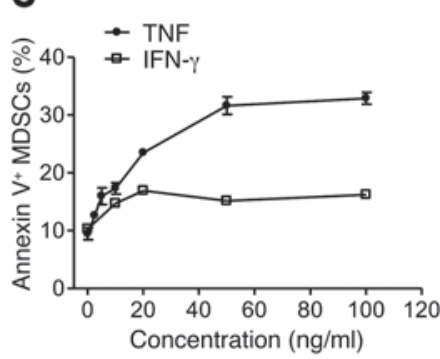

$\mathbf{F}$
D $\quad$ - Isotype ctrl $\quad$ IR $+\alpha$ PD-L1

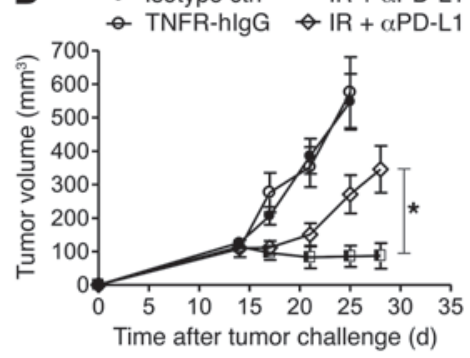

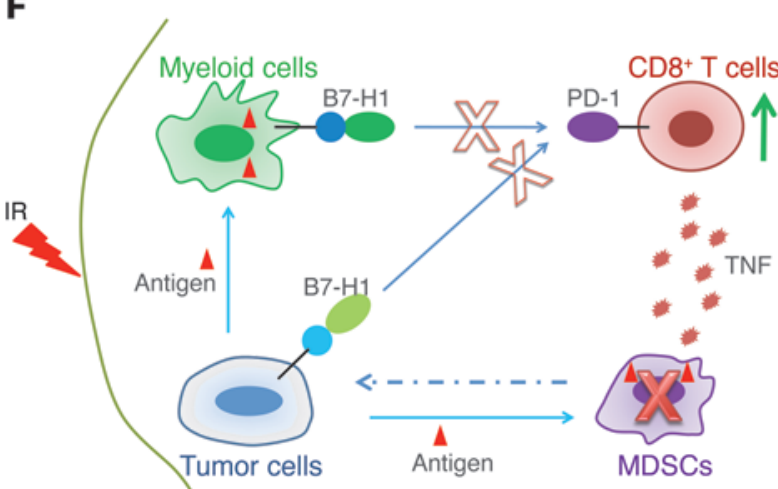

\section{Figure 6}

$\mathrm{CD}^{+} \mathrm{T}$ cells induce the apoptosis of MDSCs through TNF- $\alpha$ following combination therapy. $(\mathbf{A}$ and $\mathbf{B})$ Isolated CD8 ${ }^{+} \mathrm{T}$ cells derived from naive $\mathrm{BALB} / \mathrm{c}$ mice were stimulated with anti-CD3 and anti-CD28 for 6 hours. Then, Gr1+ cells purified from the spleen of TUBO-bearing mice were added for an additional 21 hours' coincubation. Representative dot plots $(\mathbf{A})$ and the percentage $(\mathbf{B})$ of annexin $\mathrm{V}^{+}$in $\mathrm{MDSC}$ are shown. Cells were gated on a CD11b+Ly-6C+ cell population. ${ }^{* *} P<0.01 ;{ }^{* *} P<0.001$. (C) Percentage of annexin $V^{+}$in MDSCs after treatment with increasing concentrations of TNF. Gr1 ${ }^{+}$cells purified from the spleen of TUBO-bearing mice were treated with different concentrations of TNF or IFN- $\gamma$ for 21 hours. (D) The inhibition of tumor growth with combination therapy was abrogated after the treatment of TNFR-hlgG. TUBO tumor-bearing mice were treated with IR and antibodies as described in Figure 2A. $500 \mu \mathrm{g}$ TNFR-hlgG (etanercept) was administered every 4 days for a total of three times starting on the first day of IR. ${ }^{*} P<0.05$. (E) Depletion of MDSCs augmented the efficacy of IR treatment. C57BL/ 6 mice were injected s.c. on day 0 with $1 \times 10^{6}$ MC38 cells. Tumors received 20 Gy on day 9 , and $300 \mu \mathrm{g}$ of depletion antibody against MDSCs (clone $1 \mathrm{~A} 8$ ) was administered every 2 days for a total of four times starting 1 day prior to IR. ${ }^{* \star} P<0.01$. Representative data are shown from two (A-E) experiments conducted with 4 (D) or 5 (E) mice per group. (F) Schematic of proposed mechanism for tumor destruction induced by IR and PD-L1 blockade.

A patient case report of ipilimumab and RT combination treatment correlated a reduction of MDSCs in the peripheral blood with the abscopal effect (10). These clinical discoveries support that the observations in our study could be relevant to human tumors. Our findings demonstrate that IR increases PD-L1 expression and that immune checkpoints are likely an important part of the complex regulatory milieu in the IR tumor microenvironment that suppresses antitumor immunity. Our study demonstrates that the combination of IR and anti-PD-L1 enhances host antitumor immunity and increases the efficacy of either treatment alone. Our results identify what we believe to be a novel functional link between PD-L1/PD-1 signaling and MDSCs (Figure 6F).
The $\mathrm{CD}^{+} \mathrm{T}$ cell population contains effector $\mathrm{T}$ cells and Tregs, which can function as immune stimulators and immune suppressors, respectively. Previous studies reported that $\mathrm{CD}^{+} \mathrm{T}$ cells can mediate cytotoxic function against tumor cells $(37,38)$. However, in our model, $\mathrm{CD}^{+} \mathrm{T}$ cells were dispensable for the combination of IR and anti-PD-L1 (Supplemental Figure 1). Notably, CD4 ${ }^{+-}$ Foxp $3^{+}$cells account for approximately $30 \%$ of total CD $4^{+} \mathrm{T}$ cells in tumors. Our interpretation of the dispensable nature of $\mathrm{CD}^{+}$ $\mathrm{T}$ cells is likely confounded by the capacity of anti-CD 4 antibody treatment to deplete both effector $\mathrm{CD}^{+} \mathrm{T}$ cells and regulatory $\mathrm{CD}^{+} \mathrm{T}$ cells, given the presumed opposing roles of each. Blockade of PD-1 signaling on Tregs has yielded inconsistent results, 
with either the promotion of Treg development or the reversal of their suppressive function $(39,40)$. These conflicting observations are likely a result of differences in the microenvironment or in the development of Tregs. In contrast, we found in our model that there was no significant difference in the ratio of $\mathrm{CD} 8^{+} \mathrm{T} /$ Tregs in tumors following combination treatment (Supplemental Figure 3 ), indicating that, in contrast to MDSCs, local radiation does not modulate the local population of Tregs.

MDSCs can suppress immune responses and facilitate tumor progression. We found that MDSCs expressed a high level of PD-L1, suggesting that PD-L1 might be a key mediator of MDSCmediated $\mathrm{T}$ cell suppression. Due to a lack of availability of PD-L1 conditional knockout mice, it remains impossible to determine the degree to which PD-L1 contributes to the suppressive capacity of MDSCs in vivo. We found that combination treatment of IR and anti-PD-L1 resulted in a dramatic elimination of MDSCs from the tumor microenvironment. Although the mechanisms of how MDSCs inhibit T cell activation have been well elaborated (34), the those of the reverse interaction remain undefined. We note that other mechanisms, such as FAS/FASL interactions, might be involved in the reduction of MDSCs (7). Further experiments are needed to determine whether the FAS/FASL pathway also plays a role in our model. However, we demonstrate that TNF alone is sufficient to mediate MDSC death in the absence of T cells. Furthermore, the reduction in MDSCs following combination therapy was $\mathrm{CD}^{+} \mathrm{T}$ cell dependent and mediated by TNF, which may be derived from activated $T$ cells. Our results substantiate this by indicating that TNF blockade counteracts the effect of combination treatment. Depletion of MDSCs greatly enhanced the efficacy of radiation alone, recapitulating the efficacy of combination treatment. In our model, TNF-mediated cytotoxicity was necessary and sufficient to induce cell death and eliminate MDSCs. Paradoxically, recent studies reported that TNF plays an important role in facilitating the differentiation and survival of MDSCs $(41,42)$. There are several potential explanations for the conflicting effects of TNF on MDSCs. First, TNF is likely to exert differential effects on MDSCs, depending on the developmental stage and phenotype. Second, TNF is pleiotropic and able to induce a variety of cellular responses, including inflammatory cytokine production, cell survival, cell proliferation, and cell death, depending on the timing and concentration (43). Third, the tumor microenvironment is altered with IR plus anti-PD-L1 treatment, which likely alters the local cytokine milieu and therefore the contextual nature of TNF signaling. Together, it is very likely that TNF could exert opposite effects on MDSCs during different stages of tumor development.

In summary, our work describes a previously uncharacterized mechanism by which PD-L1 blockade enhances IR. We found that the combination of IR and anti-PD-L1 treatment stimulated CD8 ${ }^{+}$ $\mathrm{T}$ cell responses, which reduced the local accumulation of MDSCs through TNF to optimize the tumor immune microenvironment and resulted in tumor regression. The importance of manipulating MDSCs in the tumor microenvironment should be evaluated in the clinical application of cancer immunotherapies. Moreover, our findings could broaden the scope of current endeavors to manipulate the immunosuppressive tumor environment and provide insight into the design of new therapeutics.

\section{Methods}

Mice and cell lines. Six- to 8-week old BALB/c mice and C57BL/6 mice were purchased from Harlan. All mice were maintained under specific patho- gen-free conditions. TUBO was cloned from a spontaneous mammary tumor in a BALB-neu Tg mouse. MC38 is a colon adenocarcinoma cell line. $3 \mathrm{~T} 3 \mathrm{~KB}$ (containing $\mathrm{H} 2-\mathrm{K}^{\mathrm{d}}$ and $\mathrm{B} 7.1$ ) and $3 \mathrm{~T} 3 \mathrm{NKB}$ (containing $\mathrm{H} 2-\mathrm{K}^{\mathrm{d}}, \mathrm{B} 7.1$ and neu) were a gift from Wei-Zen Wei (Wayne State University, Detroit, Michigan, USA).

Tumor growth and treatments. TUBO or MC38 tumor cells $\left(1 \times 10^{6}\right)$ were injected s.c. into the flanks of mice. TUBO and MC38 were allowed to grow for about 2 weeks and 8 days, respectively. Tumor volumes were measured along three orthogonal axes (a, b, and c) and calculated as tumor volume $=a b c / 2$. Tumors were treated by local IR as described previously, and tumor volumes were measured twice weekly. For $\mathrm{CD}^{+} \mathrm{T}$ cell depletion experiments, $250 \mu \mathrm{g}$ anti-CD8 (clone 2.43; Bio-XCell) per mouse was delivered four times by i.p. injection every 3 days. For the PD-L1 blockade experiment, $200 \mu \mathrm{g}$ anti-PD-L1 (clone 10F.9G2; Bio-XCell) was administered i.p. to mice every 3 days for a total of four times. For the TNF blockade experiment, $500 \mu \mathrm{g}$ TNFR-hIgG (etanercept) or isotype control was administered i.p. every 4 days for a total of three times. The MDSC depletion experiment was carried out using $300 \mu \mathrm{g}$ depletion antibody (clone 1A8; Bio-XCell), administered every 2 days for a total four times. All antibody treatments were started from the day of IR or 1 day before IR.

ELISPOT assay. Draining LNs were removed to obtain single-cell suspensions. A 96-well HTS-IP plate (Millipore) was precoated with $5 \mu \mathrm{g} / \mathrm{ml}$ anti-IFN- $\gamma$ antibody (clone R4-6A2; BD Pharmingen) overnight at $4^{\circ} \mathrm{C}$. $1 \times 10^{5}$ to $3 \times 10^{5} \mathrm{LN}$ cells were added with $3 \mathrm{~T} 3 \mathrm{NKB}$ cells at a ratio of 10:1. 3T3KB cells were used as a control cell line. After 2 days of incubation, cells were removed, $4 \mu \mathrm{g} / \mathrm{ml}$ biotinylated anti-IFN- $\gamma$ antibody (clone XMG1.2; BD Pharmingen) was added, and the plate was incubated for 2 hours at $37^{\circ} \mathrm{C}$. Avidin-horseradish peroxidase $(0.9 \mu \mathrm{g} / \mathrm{ml}$; $\mathrm{BD}$ Pharmingen) was then added, and the plate was incubated for 45 minutes at $37^{\circ} \mathrm{C}$. The cytokine spots were developed according to the manufacturer's protocol (Millipore).

Immunofluorescence staining. Frozen sections were thawed and fixed by ice-cold acetone for 10 minutes. The sections were incubated with a 1:200 dilution of rat anti-CD8 $\alpha$ (catalog 100701; BioLegend), then washed twice and followed by goat anti-rat Alexa Fluor 647. Sections were extensively washed and then incubated with a 1:500 dilution of biotinylated antimouse Gr1 (Ly6G/Ly-6C) (catalog 108403; BioLegend), a 1:200 dilution of CD11b-FITC (catalog 101205; BioLegend), and a 1:400 dilution of rabbit anti-cleaved caspase 3 (catalog 9661; Cell Signaling Technology). Sections were washed then probed with streptavidin Alexa Fluor 594 and donkey anti-rabbit Alexa Fluor 350. Sections were then washed and mounted using ProLong Gold reagent (Invitrogen). Images were acquired on a Hammatsu Orca ER firewire digital monochrome camera and a Zeiss Axiovert 200M microscope and scanned using a CRi Panoramic Scan Whole Slide Scanner (PerkinElmer). For quantification, 15-18 snapshots were randomly taken at $\times 40$ magnification from scanned wholeslide images, avoiding major vessel structures. Distances between cells were measured using Fiji/ImageJ software (W.S. Rasband, ImageJ, NIH, http://rsb.info.nih.gov/ij/, 1997-2009).

MDSCs stimulation. For cell isolation, $\mathrm{CD}^{+} \mathrm{T}$ cells from the spleen and LNs of naive mice were purified with mouse $\mathrm{CD}^{+} \mathrm{T}$ cell-positive selection kits (EasySep; STEMCELL Technologies), and Gr1+ cells from TUBO-bearing mice were purified with anti-Gr1 biotin and antibiotin beads (MACS; Miltenyi Biotec). For cell coculture experiments, $8 \times 10^{5}$ $\mathrm{CD}^{+} \mathrm{T}$ cells were stimulated with $10 \mu \mathrm{g} / \mathrm{ml}$ coated anti-CD3 and $2 \mu \mathrm{g} / \mathrm{ml}$ soluble anti-CD28 in 96-well flat-bottom plates for 6 hours, and then $4 \times 10^{5}$ $\mathrm{Gr} 1^{+}$cells were added and cocultured for an additional 21 hours in the presence of $20 \mu \mathrm{g} / \mathrm{ml}$ anti-PD-L1, $40 \mu \mathrm{g} / \mathrm{ml}$ anti-TNF- $\alpha$ (clone XT3.11; Bio-XCell), or $40 \mu \mathrm{g} / \mathrm{ml}$ anti-IFN- $\gamma$ (clone XMG1.2; Bio-XCell). For cytokine stimulation, $4 \times 10^{5} \mathrm{Gr} 1^{+}$cells were stimulated for 21 hours 
with different concentrations of murine TNF and murine IFN- $\gamma$ in 96-well plates in triplicate.

Flow cytometry. To obtain single-cell suspensions, tumor tissues were digested by $1 \mathrm{mg} / \mathrm{ml}$ collagenase IV (Sigma-Aldrich) and $0.2 \mathrm{mg} / \mathrm{ml}$ DNase I (Sigma-Aldrich) for 45 minutes at $37^{\circ} \mathrm{C}$. Cells were blocked with anti-FcR (clone 2.4G2; Bio-Xcell) and then stained with antibodies against PD-L1, PD-1, CD11b, Gr1, F4/80, CD11c, CD8, CD4, Foxp3, and CD45 (BioLegend). For apoptosis assays, MDSCs were harvested, blockaded with anti$\mathrm{FcR}$, and stained with antibodies against Ly6C, CD11b, and annexin V. Samples were collected on a FACSCalibur Flow Cytometer (BD), and data were analyzed using FlowJo software (Tree Star Inc.).

Statistics. Data were analyzed using Prism 5.0 software (GraphPad Software). Experiments were repeated two or three times. Data are represented as the mean \pm SEM for all figure panels in which error bars are shown. The $P$ values were assessed using 2 -tailed unpaired Student $t$ tests. A $P$ value of less than 0.05 was considered statistically significant.

Study approval. All studies performed on mice were approved by the IACUC of the University of Chicago.

1. Apetoh L, et al. Toll-like receptor 4-dependent contribution of the immune system to anticancer chemotherapy and radiotherapy. Nat Med. 2007; 13(9):1050-1059

2. Burnette BC, et al. The efficacy of radiotherapy relies upon induction of type $\mathrm{i}$ interferon-dependent innate and adaptive immunity. Cancer Res. 2011; 71(7):2488-2496

3. Lee $Y$, et al. Therapeutic effects of ablative radiation on local tumor require CD8+ T cells: changing strategies for cancer treatment. Blood. 2009; 114(3):589-595.

4. Barcellos-Hoff MH, Park C, Wright EG. Radiation and the microenvironment - tumorigenesis and therapy. Nat Rev Cancer. 2005;5(11):867-875.

5. Fridman WH, Pages F, Sautes-Fridman C, Galon J. The immune contexture in human tumours: impact on clinical outcome. Nat Rev Cancer. 2012; 12(4):298-306.

6. Acharyya S, et al. A CXCL1 paracrine network links cancer chemoresistance and metastasis. Cell. 2012; 150(1):165-178.

7. Sinha P, Chornoguz O, Clements VK, Artemenko KA, Zubarev RA, Ostrand-Rosenberg S. Myeloid-derived suppressor cells express the death receptor Fas and apoptose in response to T cellexpressed FasL. Blood. 2011;117(20):5381-5390.

8. Curran MA, Montalvo W, Yagita H, Allison JP. PD- 1 and CTLA- 4 combination blockade expands infiltrating $\mathrm{T}$ cells and reduces regulatory $\mathrm{T}$ and myeloid cells within B16 melanoma tumors. Proc Natl Acad Sci U S A. 2010;107(9):4275-4280.

9. Duraiswamy J, Freeman GJ, Coukos G. Therapeutic PD-1 pathway blockade augments with other modalities of immunotherapy T-cell function to prevent immune decline in ovarian cancer. Cancer Res. 2013;73(23):6900-6912.

10. Postow MA, et al. Immunologic correlates of the abscopal effect in a patient with melanoma. $N$ Engl JMed. 2012;366(10):925-931.

11. Pardoll DM. The blockade of immune checkpoints in cancer immunotherapy. Nat Rev Cancer. 2012; 12(4):252-264.

12. Liang SC, et al. Regulation of PD-1, PD-L1, and $\mathrm{PD}-\mathrm{L} 2$ expression during normal and autoimmune responses. Eur J Immunol. 2003;33(10):2706-2716.

13. Dong H, et al. Tumor-associated B7-H1 promotes T-cell apoptosis: a potential mechanism of immune evasion. Nat Med. 2002;8(8):793-800.

14. Hirahara K, et al. Interleukin-27 priming of T cells controls IL-17 production in trans via induction of the ligand PD-L1. Immunity. 2012;36(6):1017-1030.

\section{Acknowledgments}

The authors acknowledge Xuanming Yang, Eric Mortenson, and Ping Yu for their helpful scientific discussions. This research was supported in part by NIH grants CA141975 and CA97296 (to Y.X. Fu) and CA111423 (to R.R. Weichselbaum), as well as by a grant from the Ludwig Foundation (to R.R. Weichselbaum) and by a generous gift from The Foglia Foundation (to Y.X. Fu, and R.R. Weichselbaum). We thank Bristol-Myers Squibb for their support and antibodies.

Received for publication September 23, 2013, and accepted in revised form October 24, 2013.

Address correspondence to: Ralph R. Weichselbaum, $5758 \mathrm{~S}$. Maryland Avenue, MC 9006, Chicago, Illinois 60637, USA. Phone: 773.702.0817; Fax: 773.834.7233; E-mail: rrw@radonc.uchicago.edu. Or to: Yang-Xin Fu, 924 E. 57th St., BSLC R112, Chicago, Illinois 60637, USA. Phone: 773.702.0929; Fax: 773.834.8940; E-mail: yfu@uchicago.edu.

15. Spranger S, et al. Up-regulation of PD-L1, IDO, and Tregs in the melanoma tumor microenvironment is driven by $\mathrm{CD}^{+} \mathrm{T}$ cells. Sci Transl Med. 2013; 5(200):200ra116.

16. Ansari MJ, et al. The programmed death-1 (PD-1) pathway regulates autoimmune diabetes in nonobese diabetic (NOD) mice. J Exp Med. 2003; 198(1):63-69

17. Salama AD, et al. Critical role of the programmed death-1 (PD-1) pathway in regulation of experimental autoimmune encephalomyelitis. J Exp Med. 2003; 198(1):71-78

18. Barber DL, et al. Restoring function in exhausted CD8 T cells during chronic viral infection. Nature. 2006;439(7077):682-687.

19. Day CL, et al. PD-1 expression on HIV-specific T cells is associated with T-cell exhaustion and disease progression. Nature. 2006;443(7109):350-354.

20. Trautmann L, et al. Upregulation of PD-1 expression on HIV-specific CD8+ T cells leads to reversible immune dysfunction. Nat Med. 2006; 12(10):1198-1202.

21. Grivennikov SI, Greten FR, Karin M. Immunity, inflammation, and cancer. Cell. 2010;140(6):883-899.

22. Zou W, Chen L. Inhibitory B7-family molecules in the tumour microenvironment. Nat Rev Immunol. 2008;8(6):467-477.

23. Sakuishi K, Apetoh L, Sullivan JM, Blazar BR, Kuchroo VK, Anderson AC. Targeting Tim-3 and PD-1 pathways to reverse $T$ cell exhaustion and restore anti-tumor immunity. J Exp Med. 2010; 207(10):2187-2194.

24. Brahmer JR, et al. Safety and activity of anti-PD-L1 antibody in patients with advanced cancer. $N$ EnglJ Med. 2012;366(26):2455-2465.

25. Topalian SL, et al. Safety, activity, and immune correlates of anti-PD-1 antibody in cancer. $N$ Engl JMed. 2012;366(26):2443-2454.

26. Ribas A. Tumor immunotherapy directed at PD-1. N Engl J Med. 2012;366(26):2517-2519.

27. Thompson RH, et al. Costimulatory B7-H1 in renal cell carcinoma patients: Indicator of tumor aggressiveness and potential therapeutic target. Proc Natl Acad Sci U S A. 2004;101(49):17174-17179.

28. Ghebeh H, et al. The B7-H1 (PD-L1) T lymphocyte-inhibitory molecule is expressed in breast cancer patients with infiltrating ductal carcinoma: correlation with important high-risk prognostic factors. Neoplasia. 2006;8(3):190-198.

29. Kingsley DP. An interesting case of possible abscopal effect in malignant melanoma. BrJ Radiol. 1975; 48(574):863-866.
30. Wersall PJ, Blomgren H, Pisa P, Lax I, Kalkner KM, Svedman C. Regression of non-irradiated metastases after extracranial stereotactic radiotherapy in metastatic renal cell carcinoma. Acta Oncol. 2006; 45(4):493-497.

31. Liang $H$, et al. Radiation-induced equilibrium is a balance between tumor cell proliferation and T cell-mediated killing. J Immunol. 2013; 190(11):5874-5881.

32. Park $S$, et al. The therapeutic effect of anti-HER2/ neu antibody depends on both innate and adaptive immunity. Cancer Cell. 2010;18(2):160-170.

33. Xu J, et al. CSF1R signaling blockade stanches tumor-infiltrating myeloid cells and improves the efficacy of radiotherapy in prostate cancer. Cancer Res. 2013;73(9):2782-2794.

34. Gabrilovich DI, Ostrand-Rosenberg S, Bronte V. Coordinated regulation of myeloid cells by tumours. Nat Rev Immunol. 2012;12(4):253-268.

35. Corbin KS, Hellman S, Weichselbaum RR. Extracranial oligometastases: a subset of metastases curable with stereotactic radiotherapy. J Clin Oncol. 2013; 31(11):1384-1390.

36. Liauw SL, Connell PP, Weichselbaum RR. New paradigms and future challenges in radiation oncology: an update of biological targets and technology. Sci Transl Med. 2013;5(173):173sr2.

37. Quezada SA, et al. Tumor-reactive CD4(+) T cells develop cytotoxic activity and eradicate large established melanoma after transfer into lymphopenic hosts. J Exp Med. 2010;207(3):637-650.

38. Perez-Diez A, et al. CD4 cells can be more efficient at tumor rejection than CD8 cells. Blood. 2007; 109(12):5346-5354.

39. Francisco LM, et al. PD-L1 regulates the development, maintenance, and function of induced regulatory T cells. J Exp Med. 2009;206(13):3015-3029.

40. Franceschini $D$, et al. PD-L1 negatively regulates $\mathrm{CD}^{+} \mathrm{CD} 25^{+} \mathrm{Foxp}^{++}$Tregs by limiting STAT-5 phosphorylation in patients chronically infected with HCV.J Clin Invest. 2009;119(3):551-564.

41. Zhao X, et al. TNF signaling drives myeloid-derived suppressor cell accumulation. J Clin Invest. 2012; 122(11):4094-4104.

42. Sade-Feldman M, Kanterman J, Ish-Shalom E, Elnekave M, Horwitz E, Baniyash M. Tumor necrosis factor-alpha blocks differentiation and enhances suppressive activity of immature myeloid cells during chronic inflammation. Immunity. 2013; 38(3):541-554.

43. Varfolomeev EE, Ashkenazi A. Tumor necrosis factor: an apoptosis JuNKie? Cell. 2004;116(4):491-497. 